\title{
Complex Neuro-Cognitive Systems
}

\author{
Andreas Schierwagen \\ Institute for Computer Science, Intelligent Systems Department, \\ University of Leipzig, Leipzig, Germany \\ schierwa@informatik. uni-leipzig.de \\ http://www.informatik. uni-leipzig.de/ ${ }^{\sim}$ schierwa
}

\begin{abstract}
Cognitive functions such as a perception, thinking and acting are based on the working of the brain, one of the most complex systems we know. The traditional scientific methodology, however, has proved to be not sufficient to understand the relation between brain and cognition. The aim of this paper is to review an alternative methodology - nonlinear dynamical analysis - and to demonstrate its benefit for cognitive neuroscience in cases when the usual reductionist method fails.
\end{abstract}

\section{Introduction}

Cognitive science aims at both understanding natural cognition (as in humans or animals) and creating artificial systems resembling the natural original. There are basically two established ways of doing research in cognitive science (including cognitive and computational neuroscience), either top-down or bottom-up. Both approaches have their pro's and con's, and none is practised in the pure form. For example, the main research strategy in computational neuroscience - reverse engineering - is basically bottom-up. It is informed, however, by topdown considerations about the goal of the computation performed by the neural system under study. It is expected that united efforts of this kind will succeed in providing a theory of cognition that is ultimately grounded in brain processes.

However, objections have been also raised saying that the established methods do not meet the complexity of the object of study, and that the research methodolgy must be complemented accordingly. In this paper, I'll first describe briefly the two traditional ways of doing cognitive (neuro-)science that are commonly thought to exhaust the possibilities. Then a third way of analysis - nonlinear dynamical analysis - is described that relies on results of complexity research, i.e. chaos theory and nonlinear modeling.

\section{Top-Down and Bottom-Up Approaches}

The top-down approach consists of (1) specifying a cognitive function by focusing on the characterization of the abstract principles that underlie that function. Ideally, it proposes (2) possible neural algorithms that might subserve this cognitive function, and finally (3) maps these algorithms onto brain circuits. In many

J.M. Ferrández et al. (Eds.): IWINAC 2011, Part I, LNCS 6686, pp. 1-9, 2011.

(C) Springer-Verlag Berlin Heidelberg 2011 
cases, however, the identification steps (2) and (3)have proved to be very difficult or unfeasible. The bottom-up approach consists of describing the structural and functional properties of given brain circuits, and then bringing this function into congruence with the cognitive function under study.

Previously [12, I have shown that both approaches set up the method of reverse engineering. This method combines analysis with synthesis in the following way. Analysis is carried out top-down by specifying first a certain cognitive function which is assumed to be computed through the cortex or some cortical subsystem. Then a decompositional analysis is performed, i.e. the cortical system is both functionally (computationally) and structurally decomposed, and the interactions between components are determined. Following the localisation concept, the functional components (computational units) are assigned to the anatomical components.

Synthesis first requires modelling, i.e building a structurally adequate, functional model of the computational unit. Based on knowledge of localised components and their interactions, a structurally adequate network model of the cortical system is build composed of the computational unit models. Simulations of the network model should eventually prove that the specific cognitive function under study is generated this way.

Recent efforts to build artificial brain 1 employ both approaches [6 7]. Largescale brain simulations attempt to model in a realistic fashion the details of the brain organisation, i.e. its structure and function. The Blue Brain Project is one prominent example of this bottom-up modelling strategy; its explicit goal is to reverse engineer the brain. On the other hand, biologically inspired cognitive architectures (BICAs) rely on the top-down approach. They attempt to achieve the brain's cognitive functionality by emulating its high-level performance without capturing the neural details.

In the survey 677] it was concluded that the two approaches display very different strengths. While bottom-up brain simulations are confined to syntactic aspects like how collections of neurons synchronize their electrical discharges, they do not tell anything about semantics, i.e. how brain processes enable cognitive agents to achieve goals, select actions or process information. In contrast, BICAs propose how brains may realise cognitive functions, but as yet they demonstrate rather simplistic behaviour compared to real brains. The authors conjecture that the deficiencies may be due to the fact that "BICAs lack the chaotic, complex generativity that comes from neural nonlinear dynamics - i.e. they have the sensible and brain-like higher-level structures, but lack the lower-level complexity and emergence that one sees in large-scale brain simulations." [7, p. 48]. Bringing large-scale brain simulations and BICAs together, they suggest, will accomplish progress toward the goals of cognitive science - understanding the brain and creating artificial cognitive systems.

\footnotetext{
1 "Artificial brain" is a term used to describe research that aims to develop software and hardware with cognitive abilities similar to humans or other mammals. Prominent examples are SyNAPSE (Systems of Neuromorphic Adaptive Plastic Scalable Electronics) 3], the Blue Brain Project [4] and the China Brain Project [5].
} 


\section{Complex Systems}

The suggestion to integrate bottom-up, large-scale brain simulations and topdown theories such as BICAs to progress in neuro-cognition research has been made from time to time. However, the predicted success has not appeared what is apparently due to the fact that both approaches have restrictions which cannot be overcome even by integration. Actually, both analysis methods are applicable only to a limited class of systems, the (near-)decomposable systems, as shown elsewhere 122 . There I have argued that the subjects of study of cognitive and computational neuroscience - cognitive systems that realise functions localised in neural circuits of the brain - are not members of this class. They are instances of complex systems which resist the usual reductionist analyses.

The study of complex systems originated during the last three decades or so from the interplay of disciplines such as physics, mathematics, biology, economy, engineering, and computer science. There is still no generally accepted definition of complexity, despite a multitude of proposed approaches (e.g. 8910]).

Important is the following distinction: we must differentiate between systems that are complex and those which are merely complicated [11, p.511]:

A complicated system is composed of a large number of interacting components. Importantly, the properties of such a system can be accurately predicted from a knowledge of the properties of each of its components and a complete enumeration of their interactions. In other words, a complicated system is exactly the sum of its parts. Complex, on the other hand, is a term reserved for systems that display properties that are not predictable from a complete description of their components, and that are generally considered to be qualitatively different from the sum of their parts.

Editorial. Nature Biotechnology, 1999

From complexity theory we know that complex phenomena can be produced from the interaction of rather simple components. Well-understood examples are artificial neural networks and cellular automata. These are compositionally complex systems, and it is indeed feasible to predict their behaviour from the knowledge of the properties of the components and their interactions. This is completely different in the case of complex systems whose behaviour emerges in an unpredictable way. The question then arises: how should complex systems be studied, and which methods of investigation are available? In the following, I will consider the method of nonlinear dynamical analysis. Other methods include relational modelling [1213] and quantum theories [14].

\section{Nonlinear Dynamical Analysis}

\subsection{Terminology}

To apply a nonlinear dynamical analysis to a complex system means to specify its temporal evolution. The state space (or phase space) of the system consists of 
the set of all its possible states, $s$, determined by the values of all the variables that describe the system at a particular moment in time. If $s$ is described by $m$ variables, it can be represented by a point in $m$-dimensional space, $s \in R^{m}$. The system dynamics is given by the set of equations or rules that control the system evolution over time. In many cases the dynamics consists of a system of $m$ coupled differential equations, one for each system variable. Most natural systems are continuous systems and therefore $s$ is a function of time, $s(t)$. As the system evolves in time its states trace a trajectory in the state space.

An attractor in state space may be defined as a state (point attractor) or set of states, toward which the system settles (relaxes) over time. Besides point attractors, three more attractor types can occur: (i) limit cycles; (ii) torus attractors; (iii) chaotic attractors. A limit cycle is a closed trajectory (an orbit) in state space that the system performs cyclically; when a system evolves towards a periodic attractor, it will oscillate endless through the same sequence of states (unless perturbed). A torus attractor has a 'donut like' shape, and corresponds to quasi periodic dynamics. A chaotic attractor is a non-repeating orbit in state space, i.e. the system dynamics, although deterministic, will never repeat the same state; it is called deterministic chaos.

Several measures are used to characterize the properties of attractors, and thus of the corresponding dynamics more exactly. Correlation dimension is a measure of the complexity of the deterministic dynamics. A point attractor has dimension zero, a limit cycle dimension one, a torus has an integer dimension corresponding to the number of superimposed periodic oscillations, and a chaotic attractor has a fractal dimension. Lyapunov exponents indicate the exponential divergence (positive exponents) or convergence (negative exponents) of nearby trajectories on the attractor, thus giving information about the systems dependence on initial conditions. A positive Lyapunov exponent is a strong indicator of chaos.

\subsection{An Example}

Complex behaviour (dynamical complexity) can arise even in simple systems with low compositional complexity. The damped, periodically driven non-linear
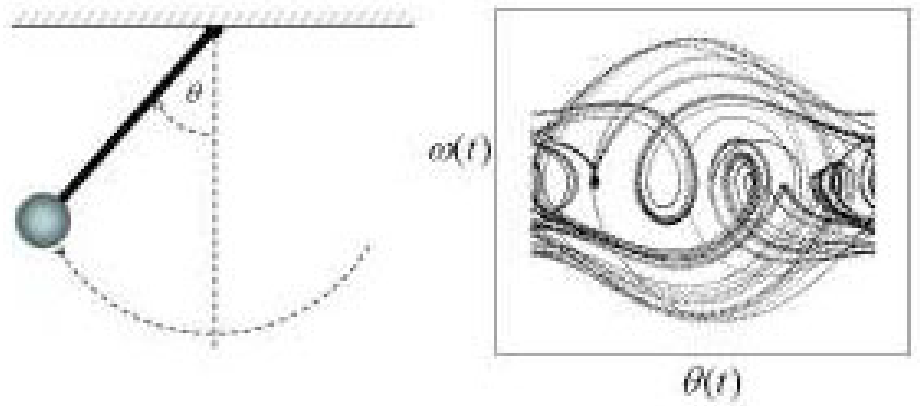

Fig. 1. The damped, periodically driven non-linear pendulum. Displayed is the chaotic attractor in the $(\theta, \omega)$-phase space that occurs for large values of the driving force $g$. 
pendulum is suited for illustrating the principles behind complex dynamics and chaotic attractors (Fig. 22). The dimensionless motion equation of the pendulum is:

$$
\frac{d^{2} \theta}{d t^{2}}+\frac{1}{q} \frac{d \theta}{d t}+\sin \theta=g \cos \left(\omega_{D} t\right)
$$

with $\theta$ the angular position in radians, $q$ the damping parameter, $g$ the amplitude of the driving force, and $\omega_{D}$ the frequency of that force. For small angle $\theta$ the equation can be integrated, i.e. the pendulum either undergoes regular oscillations or, without a driving term, eventually stop swinging. For larger angles $\theta$, however, this approximation is invalid and hence the equation can no longer be solved analytically.

The dynamical variables of the system, i.e. the angular position $\theta$ and velocity $\omega=d \theta / d t$, are the coordinates defining the system's phase space. In the twodimensional case, the variables can be plotted to display a phase portrait of the dynamical behaviour.

By varying the parameters $q, g$ and $\omega_{D}$ of the motion equation (11) and then plotting the resulting phase portrait a wide range of behaviour can be observed. In the case of a non-zero damping parameter and no driving force to replace the energy loss, the pendulum is a dissipative system, i.e. it comes to the resting point $(0,0)$, a fixpoint attractor. Using a non-zero driving force $g$, the attractor is no longer a single point at $(0,0)$ but now a closed orbit, that is, the pendulum undergoes regular motion. A bifurcation has occurred, changing the fixpoint into a limit cycle attractor. Increasing the driving force $g$ further, a sequence of period doublings occurs which continues as $g$ is increased until a point is reached where the motion of the pendulum ceases to be regular and becomes chaotic, i.e. a chaotic attractor occurred.

This example nicely shows that even in a compositionally very simple system the dynamics can be chaotic. In this case, the analysis could be made because the dynamical system model, i.e. the equation of motion (1), is known.

\subsection{Nonlinear Time Series Analysis}

We have discussed complex systems with nonlinear dynamics so far using the bottom-up, deductive approach: given the system equations, the behaviour of the system can be predicted. However, the situation in cognitive neuroscience is totally different: instead of the system equations, a set of observations is given, say in the form of an EEG record. The problem then is to find a way to get from the observations of a system with unknown properties to an understanding of the dynamics of the underlying system. This can be achieved by nonlinear time series analysis, a systemic approach starting with the output of the system, and working towards the state space, attractors and their properties.

Nonlinear time series analysis proceeds as follows: (i) reconstruction of the systems dynamics in state space; (ii) characterization of the reconstructed attractor; (iii) checking the validity of the procedure [17. 
State space reconstruction. In order to reconstruct the state space using a time series, it is resolved into coordinate values of a $d$-dimensional embedding space by an embedding method. Let the state space be characterized by the set of variables, $\left\{x_{0}(t), x_{1}(t), \ldots, x_{d-1}(t)\right\}$. Most frequently used is time delay embedding. Assume that only time series $x_{0}(t)$ is available 2 . Then time-delayed values of this series are used,

$$
\left\{x_{0}(t), x_{0}(t+\tau), \ldots, x_{0}[t+(d-1) \tau]\right\} .
$$

This set is topologically equivalent to the original set of system variables, see [18. These variables are obtained by shifting the original time series by a fixed time lag $\tau=m \Delta t$ where $m$ is an integer and $\Delta t$ is the interval between successive samplings. A most important problem of state space reconstruction is the determination of the delay time $\tau$ and embedding dimension $d$ for which several methods are available. This result is known as Taken's famous 'Embedding Theorem' which says: valuable information about the dynamics of the system can be obtained, even if direct access to all the systems variables is impossible, as it is common in cognitive neuroscience!

Characterization of the reconstructed attractor. After reconstruction of the attractor by embedding the next step is to characterize it. A common way to do this is to visualize it with a phase portrait. A phase portrait is a twoor three-dimensional plot of the reconstructed state space and the attractor. The graph shown in Fig. 1 is an example of a two-dimensional phase portrait. Other methods to display the reconstructed trajectories are Poincaré sections and recurrence plots 19 .

Following embedding and visualization of the reconstructed attractor the next step is to attempt to characterize it in a quantitative way. The classic measures applied are correlation dimension, Lyapunov exponents and entropy mentioned in Section 4, and new measures introduced frequently in the literature.

Checking the validity of the procedure. The interpretation of nonlinear measures is known to present problems sometimes since noisy time series can give rise to the unwarranted impression of low-dimensional dynamics and chaos. Therefore, the nonlinearity of the time series should be tested. It is customary to do this by surrogate data test. The null hypothesis of the test is that the original time series is generated from a linear stochastic process (possibly undergoing a nonlinear static transform). Demonstration of nonlinearity is important since only nonlinear dynamical systems can have attractors other than a trivial fixpoint attractor. Chaos in particular can only occur in nonlinear dynamical systems.

\section{Explanation by Nonlinear Dynamic Analysis}

In the preceding sections the methodology of nonlinear dynamic analysis was outlined roughly. We are now prepared to demonstrate how it is used in cognitive

\footnotetext{
${ }^{2} x_{0}$ may represent the electrical potential recorded by the EEG.
} 


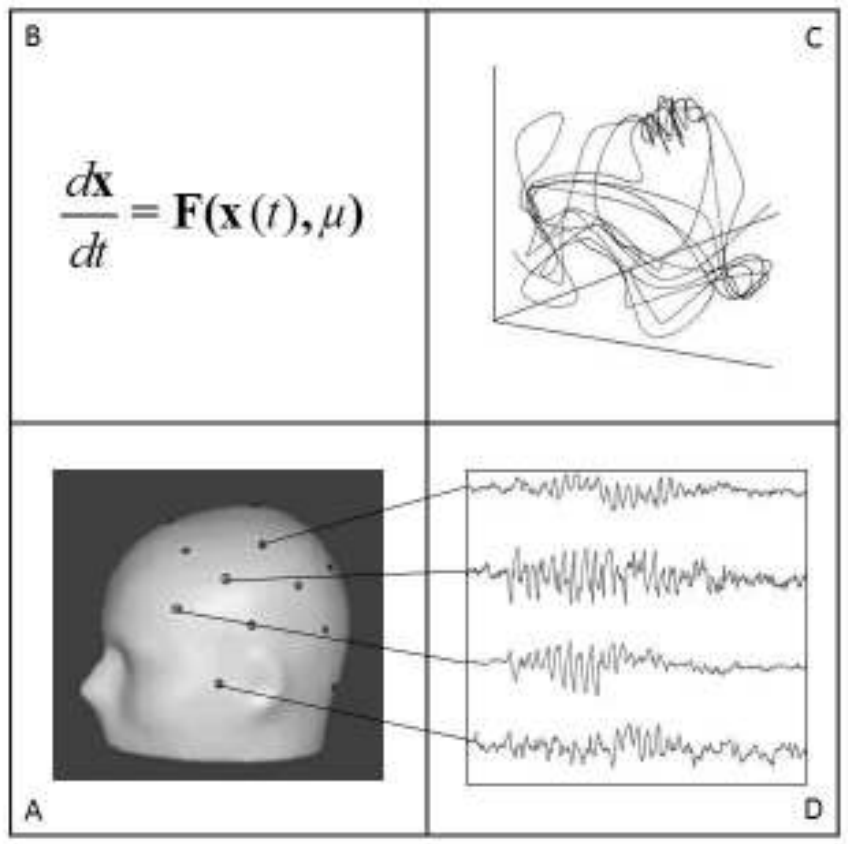

Fig. 2. Reductionist, deductive explanation is illustrated by following the path through cells $A \rightarrow B \rightarrow C \rightarrow D$. This approach fails in non-decomposable systems with complex dynamics since no system equations can be established. Instead, observable behaviour of the real system is explained by nonlinear dynamic analysis, i.e. move $C \rightarrow D$ is to be made (inspired by [16]).

neuroscience. Let us consider the situation presented in Fig. 2. In cell $A$ the physical system under study is displayed (a human's head with EEG recording sites). Cell $B$ shows a formal model (a system of nonlinear differential equations). Establishing the model requires that all the variables determining the system and their dynamic connections are exactly known. In cell $C$ the phase portrait in the system state space is schematically illustrated. It allows to describe the possible system behaviour in terms of trajectories, attractors, bifurcations etc. Cell $D$ presents the observed system behaviour which is often measured in cognitive science in the form of time series (in this case the EEG activity recorded via several channels).

The usual kind of reductionist, deductive explanation can be illustrated by following the path through cells $A \rightarrow B \rightarrow C \rightarrow D$ in Fig. 2. The behaviour of the physical system as measured at the emergent level is reduced via the formal model to the lower level of the physical substrate. The transitions between the fields are all non-trivial. Move $A \rightarrow B$ requires to determine the relevant system variables and to study their dynamics which is rather unfeasible in the case of neuro-cognitive systems. Move $B \rightarrow C$ means nonlinear dynamical analysis of system equations which may pose serious difficulties, depending on system 
characteristics. Finally, by moving $C \rightarrow D$ the formal constructs of nonlinear system theory are to be mapped on real, observable phenomena in the neurocognitive system. This move again is very challenging, and cannot be formalised 12 .

Emergent phenomena of the real, dynamically complex system must be explained using nonlinear dynamic analysis since traditional analyses fail. That is, in the scheme of Fig. 2 only move $C \rightarrow D$ is (and can be) made. A meanwhile famous example is the work by Babloyantz and Destexhe [15] who demonstrated that the epileptic electric brain activity measured by the EEG forms a chaotic attractor. Here the Lyapunov exponents of the attractors and the embedding dimension of the phase space was calculated from the time series of the EEG. This was done without having available a formal model. From the mere fact that a chaotic attractor with certain mathematical properties is present, non-trivial conclusions (e.g., on stability, dimensionality of embedding space etc.) has been drawn.

Systemic explanations of this kind have explanatory power because dynamically complex systems own certain universal properties. If a whole class of dynamic systems is characterised by a certain qualitative property, it is unnecessary to know the exact form of the special dynamic model which explains the particular emergent phenomenon. It is only necessary to assure that the system under study belongs to the corresponding class of dynamic systems in which certain qualitative phenomena are universal. This kind of the explanation works because classes of dynamically complex systems own qualitative properties (attractor types, bifurcations, pattern generation, chaos).

\section{Conclusions}

The progress made with analyses of compositionally complex (complicated) systems such as artificial neural networks, cellular automata etc. has led many to believe that this can be achieved with dynamically complex systems, too. This means that the reverse engineering method has to be applied to the observed dynamically complex phenomenon to work out which mechanism explains it best. A mechanism is known if the participating system components and their interactions are known, i.e. the term 'mechanism' is nearly synonymous with 'decomposability'. If we remember that dynamically complex systems are not decomposable, it follows that no mechanism can be revealed, and the reductionist analysis fails!

In this vein, proponents of the synthetic bottom-up simulation approach often argue that a systemic, non-reductionist approach such as nonlinear dynamical analysis merely provides provisional solutions which become only then full explanations when they are complemented by a formal model and by the physical basis of the empirical system. However, this view again ignores the specificity of the dynamically complex systems which do not allow reductionist, mechanistic explanation. Thus, nonlinear dynamic analyses provide full-value explanations which are highly appropriate to the situation in cognitive neuroscience. 


\section{References}

1. Schierwagen, A.: Brain complexity: analysis, models and limits of understanding. In: Mira, J., Ferrández, J.M., Álvarez, J.R., de la Paz, F., Toledo, F.J. (eds.) IWINAC 2009. LNCS, vol. 5601, pp. 195-204. Springer, Heidelberg (2009)

2. Schierwagen, A.: On reverse engineering in the cognitive and brain sciences. Natural Comput (2011) (in press)

3. Systems of Neuromorphic Adaptive Plastic Scalable Electronics (SyNAPSE). DARPA / IBM (2008)

4. Markram, H.: The Blue Brain Project. Nature Rev. Neurosci. 7, 153-160 (2006)

5. de Garis, H., et al.: The China-Brain Project: Building China's Artificial Brain Using an Evolved Neural Net Module Approach. In: Wang, P., Goertzel, B., Franklin, S. (eds.) Proceedings First AGI Conference, pp. 107-121. IOS Press, Amsterdam (2008)

6. de Garis, H., Shuoa, C., Goertzel, B., Ruiting, L.: A world survey of artificial brain projects, Part I: Large-scale brain simulations. Neurocomput. 74, 3-29 (2010)

7. Goertzel, B., Ruiting, L., Arel, I., de Garis, H., Chen, S.: World survey of artificial brains, Part II: Biologically inspired cognitive architectures. Neurocomput. 74, 30-49 (2010)

8. Edmonds, B.: Syntactic Measures of Complexity. PhD thesis, University of Manchester (1999)

9. Chu, D., Strand, R., Fjelland, R.: Theories of complexity. Complexity 8, 19-30 (2003)

10. Gershenson, C.: Complexity. arXiv:1003.5947v1

11. Editorial. Complicated is not complex. Nature Biotechnology 17, 511 (1999)

12. Rosen, R.: Life Itself: A Comprehensive Inquiry into the Nature, Origin, and Fabrication of Life. Columbia University Press, New York (1991)

13. Rosen, R.: Essays on Life Itself. Columbia University Press, New York (2000)

14. Kitto, K.: High End Complexity. Intern. J. Gen. Syst. 37, 689-714 (2008)

15. Babloyantz, A., Destexhe, A.: Low-dimensional chaos in an instance of epilepsy. Proc. Natl. Acad. Sci. USA 83, 3513-3517 (1986)

16. Jaeger, H.: Dynamische Systeme in der Kognitionswissenschaft. Kognitionswissenschaft 5, 151-174 (1996)

17. Stam, C.J.: Nonlinear dynamical analysis of EEG and MEG: Review of an emerging field. Clin. Neurophysiol. 116, 2266-2301 (2005)

18. Takens, F.: Detecting strange attractors in turbulence. Lecture Notes Math. 898, 366-381 (1981)

19. Kantz, H., Schreiber, T.: Nonlinear Time Series Analysis. Cambridge University Press, Cambridge (1997) 